\title{
Definição da Arquitetura de Informação em organismo da Administração Pública Local
}

\author{
Filipe Sá ${ }^{1}$ Álvaro Rocha ${ }^{2}$ \\ filipe@cm-penacova.pt, amrocha@ufp.edu.pt \\ ${ }^{1}$ Câmara Municipal de Penacova, Largo Alberto Leitão, 5, 3360-341 Penacova, Portugal \\ 2 Universidade Fernando Pessoa, Praça 9 de Abril, 349, 4249-004 Porto, Portugal \\ DOI: $10.4304 /$ risti.10.51-64
}

\begin{abstract}
Resumo: Uma arquitetura de informação é a base para as organizações disporem de um sistema de informação abrangente e integrado, capaz de proporcionar uma resposta alinhada com as exigências do ambiente competitivo atual. Neste artigo são identificadas metodologias representativas para a definição de arquiteturas de informação e é apresentado um estudo de planeamento da arquitetura de informação para um organismo da administração pública local, com recurso à metodologia BSP Adaptada, a qual se mostrou suficientemente flexível e adequada à concretização dos objetivos perseguidos.
\end{abstract}

Palavras-chave: Planeamento de Sistemas de Informação; Arquiteturas de Informação; Integração de Sistemas de Informação; Governo Eletrónico.

\begin{abstract}
An Information Architecture is the basis for a comprehensive and integrated information system of any organization, capable of providing answers that meet the challenges posed by the contemporary competitive environment. In this paper we identify representative methodologies used in Information Architecture definitions, and we present an Information Architecture Planning case study for a Local Public Administration organization, where we implement an Adapted BSP Methodology that is sufficiently flexible and suited to the goals we propose.
\end{abstract}

Key-words: Information Systems Planning; Information Architecture; Information Systems Integration; E-Government. 


\section{Introdução}

A globalização dos mercados, com a consequente intensificação da competitividade e o crescente nível de exigência relativamente a produtos e serviços, levam a que praticamente todos os aspetos das organizações influenciem o seu posicionamento competitivo, muito particularmente a eficácia do seu Sistema de Informação (SI) (Teixeira et al., 2012; Rocha, 2011, Huang \& Wang 2010; Mohammad, 2009).

Assim, a Administração Pública Local enfrenta um desafio no âmbito da modernização administrativa, procurando aproximar os Munícipes dos seus serviços, havendo, pois, a necessidade constante em desmaterializar os seus processos (Almeida, 2011; Torres, 2009; Rocha et al. 2005).

O Município de Penacova, pertencente ao Distrito de Coimbra, Portugal, não é exceção e existe nele essa necessidade. A informação é cada vez maior e a sua consulta tem de ser rápida e eficaz para que as decisões e ações sejam as mais eficientes possíveis.

No contexto deste trabalho, pretendeu-se elaborar um estudo com a finalidade de definir e validar uma arquitetura de informação para a Secção de Obras Particulares do Município de Penacova, capaz de responder eficiente e eficazmente às suas necessidades de informação.

Consequentemente desenvolveu-se um estudo sobre conceitos e metodologias existentes, para que a arquitetura de informação a definir fosse coesa e baseada num trabalho académico que pudesse sustentar um produto final realmente útil.

Assim, foram objetivos específicos deste trabalho:

- Analisar metodologias representativas no planeamento de arquiteturas de informação;

- Selecionar uma metodologia e aplicá-la na realidade;

- Propor e validar uma arquitetura de informação para a Secção de Obras Particulares do Município de Penacova;

- Refletir sobre as vantagens e desvantagens da metodologia aplicada.

Nas próximas secções apresenta-se a metodologia seguida no estudo, identificam-se metodologias representativas para o planeamento de arquiteturas de informação e apresenta-se o estudo de caso de definição de uma arquitetura de informação para a Secção de Obras Particulares do Município de Penacova. Por último, discutem-se os resultados, retiram-se algumas conclusões e aponta-se a direção do trabalho futuro.

\section{Metodologia do Estudo}

Numa primeira fase foi realizada uma revisão de literatura através da análise de uma amostra criteriosa de livros, dissertações, teses e artigos científicos da área, que permitiu selecionar e conhecer a metodologia de planeamento de arquiteturas de informação a aplicar no caso prático.

Na continuação da abordagem anterior, foi seguido o método estudo de casos, sendo este muito adotado em trabalhos de investigação de carácter quantitativo e qualitativo 
(Höst \& Runeson, 2008; Höst \& Runeson 2007), que orientou a aplicação da Metodologia BSP Adaptada de Amaral \& Varajão (2007), na Secção de Obras Particulares do Município de Penacova.

Com base em todos os elementos recolhidos e analisados, foi proposta e validada uma arquitetura de informação para o desenvolvimento de sistemas de informação que suportem as diferentes necessidades de informação, de forma completa e integrada, nesta Secção do Município de Penacova.

Finalmente discutiram-se os resultados e refletiu-se sobre as vantagens e desvantagens da metodologia usada.

\section{Metodologias de Planeamento de Arquiteturas de Informação}

A arquitetura da informação de uma organização envolve a definição do relacionamento de processos de negócio com as classes de dados. Isso permite a avaliação da partilha de dados dentro da organização. A arquitetura de informação também proporciona a base para a gestão de recursos e planeamento tático, que permite a implementação ordenada da arquitetura da informação.

O foco deste trabalho passou também pela identificação de metodologias capazes de ajudarem a planear a arquitetura de informação de uma organização. Foram, consequentemente, selecionadas e estudadas a Metodologia BSP - Business Systems Planning (IBM, 1984), a Metodologia BSP Adaptada (Amaral \& Varajão, 2007), a Framework de Zachman (Zachman, Inmon \& Geiger, 1997; Zachman, 1996, Zachman, 1987), a Federal Enterprise Architecture Framework (Spewak \& Hill, 1995) e a Metodologia Enterprise Architecture Planning (Sayles, 2003; CIOC, 2001).

Estas são, pois, as quatro metodologias consideradas representativas na definição de Arquiteturas de Informação, dado serem referidas, estudadas, adotadas e/ou aplicadas em vários outros trabalhos académicos (e.g., Abbas et al. 2010; Huang \& Wang 2010; Rocha \& Santos, 2010; Mohammad, 2009; Mesquita, 2007; Casagrande, 2005; Tomé, 2004; Costa, 2002; Rocha, 2002; Souza, 2001; Sakamoto \& Ball 1982).

Para aplicação no estudo de caso foi selecionada a Metodologia BSP Adaptada de Amaral \& Varajão (2007).

A sua escolha deveu-se aos seguintes fatores:

- Boa documentação de suporte;

- Por ser inspirada na Metodologia BSP original, mas apresentando-se com novos detalhes e com uma diferente organização das atividades ajustada ao contexto atual;

- Por ir ao encontro dos fatores chave no sucesso do planeamento, desenvolvimento e implementação de uma arquitetura de informação;

- A facilidade em adequar as várias fases da metodologia à realidade da Câmara Municipal de Penacova; 
- As matrizes que descrevem a arquitetura de informação permitirem com clareza e eficácia uma análise aos processos e às classes de dados, além de permitirem uma análise sobre os fluxos de informação, o que facilita uma maior compreensão do estudo global;

- Com a utilização da Matriz Aplicações/Classes de Dados, Matriz Aplicações/Processos e Matriz Aplicações/Organização, permite a identificação clara e inequívoca, do suporte que os SI atuais e futuros têm na arquitetura de informação da Secção de Obras Particulares do Município de Penacova.

\section{Estudo de Caso: Secção de Obras Particulares do Município de Penacova}

Nesta parte do artigo é descrito um estudo realizado na Secção de Obras Particulares do Município de Penacova. Este estudo passou pelo planeamento de uma arquitetura de informação, baseada na Metodologia BSP Adaptada, de Amaral \& Varajão (2007). A mesma foi adaptada à realidade do trabalho e à dimensão do estudo.

\subsection{Caracterização da Secção de Obras Particulares}

O Município de Penacova está inserido no Distrito de Coimbra. É um município que possui uma área de $220 \mathrm{Km} 2$ com 15251 habitantes - Censos 2011 - distribuídos por 11 freguesias.

A Câmara Municipal de Penacova é, pois, um organismo de Administração Pública Local. No final do ano de 2010 dispunha de 149 colaboradores. A sua Secção de Obras Particulares faz parte da Divisão de Ambiente, Serviços Urbanos e Obras Municipais e depende hierarquicamente do Presidente da Câmara, que pode delegar as competências em Vereadores ou em alguém do seu gabinete. É composta pelas subunidades orgânicas Planeamento de Serviços; Planeamento do Território; Gestão de Urbanística e Obras; Serviços Municipais de Fiscalização; e Secção Administrativa. Na divisão geral trabalham 74 funcionários, dos quais 17 estão destacados a tempo inteiro no serviço da Secção de Obras Particulares.

De uma forma genérica esta Secção de Obras Particulares tem como principal objetivo licenciar e regular todas as construções no Município de Penacova. Alguns dos processos resultam em projetos de obras, loteamentos, certidões, correspondência, emissão de alvarás, cobrança de taxas, processos de águas, saneamento, etc.

Apesar de ser uma Secção interna, interage no decorrer dos seus processos com outras secções internas, como por exemplo a Tesouraria ou a Contabilidade. Existem interações externas tanto com Munícipes como com entidades para licenciamentos, autorizações ou pedidos de pareceres.

Nesta Secção é feita a receção e respetiva movimentação de todo o expediente ligado a obras particulares. 


\subsection{Aplicação da Metodologia BSP Adaptada}

Nesta parte do artigo é descrita a aplicação da Metodologia BSP Adaptada, de Amaral \& Varajão (2007), para o planeamento de uma arquitetura de informação para a Secção de Obras Particulares da Câmara Municipal de Penacova. Esta aplicação justifica-se porque não existia nenhum estudo nem documento formal de PSI para esta Secção da Câmara Municipal e porque a mesma necessita de repensar todo o seu SI.

Consequentemente foram implementadas as fases da metodologia selecionada, embora adaptadas à realidade do objeto do estudo e ao âmbito da investigação. Foram, assim, definidos o âmbito e o objetivo do estudo, a equipa do projeto, a logística. Foi identificada a informação a reunir sobre recursos humanos, recursos financeiros, serviços prestados e clientes. Caracterizou-se o hardware, o software instalado e os sistemas de informação. Identificou-se ainda a missão, a visão, as metas e objetivos estratégicos da Secção de Obras Particulares da Câmara Municipal de Penacova. Definiram-se, também, as suas entidades. Com base nas reuniões tidas com o Chefe de Divisão da Secção de Obras e com o Presidente da Câmara Municipal, identificaram-se as entidades: Presidente; Chefe de Divisão; Técnicos; Administrativos; Fiscal Municipal; Comissão de Vistoria; e Munícipes. Este conjunto de entidades contém todos os elementos que intervêm nos processos da Secção. Definiram-se, ainda, os processos e as classes de dados.

\section{Processos}

Segundo Amaral \& Varajão (2007) os processos da organização são definidos como "grupos de decisões relacionadas logicamente" ou "atividades necessárias para gerir os recursos da organização”. Ainda de acordo com os mesmos autores, uma definição dos processos organizacionais levará a:

- Um SI independente da estrutura organizacional;

- Uma compreensão de como a organização realiza a sua missão;

- Uma base para o planeamento da Arquitetura de Informação, identificando o seu âmbito, tornando-a modular e determinando prioridades para o seu desenvolvimento;

- Uma base para a identificação de requisitos chave de dados.

Tendo em conta as regras para identificação de processos, com base nas reuniões efetuadas, foi primeiramente elaborado um esquema contendo toda a tramitação e processos existentes na Secção de Obras Particulares. Feito o levantamento dos processos, estes foram definidos, constituindo o "Dicionário de Processos". No final foi efetuada uma validação dos mesmos.

Foram identificados e descritos 37 processos. Elaborar Plano de Atividades (P1) é um exemplo dos processos identificados, sendo a sua descrição: "Conjunto de ações $e$ decisões que visam elaborar o Plano de Atividades para a Secção de Obras Particulares, definindo objetivos, responsabilidades e identificando meios para concretizá-lo.”. 


\section{Classes de Dados}

Amaral \& Varajão (2007) referem que "uma classe de dados é um agrupamento de dados relacionados com aspetos (ou entidades) que são relevantes para a informação. As classes de dados devem representar dados que precisam de estar disponíveis para a realização das atividades da organização". Baseados nestes pressupostos e posteriormente à definição do dicionário de processos, foi possível identificar e definir as classes de dados existentes na Secção e assim elaborar o dicionário de classes de dados.

Foram identificadas e descritas 42 classes de dados. Livro de Obra (C30) é um dos exemplos das classes de dados identificadas, sendo a sua descrição: "Documento que contém todo o historial da construção da obra, incluindo dados dos técnicos responsáveis, resumos diários de progressão da obra, alterações ao projeto, visitas dos fiscais municipais e autos dos fiscais municipais".

Após a identificação e a descrição do dicionário de dados dos processos e das classes de dados, determinámos a criação e uso dos dados por cada processo. Na Figura 1 apresentamos o exemplo do processo P1 (Elaborar Plano de Atividades).

\begin{tabular}{|cc|}
\hline Cria 1 - Elaborar Plano de Atividades \\
Usa \\
C1 - Plano de atividades & $\mathrm{C}_{3}$ - Folha de objetivos \\
& $\mathrm{C}_{5}$ - Mapa de Férias \\
& $\mathrm{C} 4-$ Ficha de avaliação/RH \\
\hline
\end{tabular}

Figura 1 - Criação e uso de classes de dados por processo.

\section{Definição da Arquitetura de Informação}

Após identificação, descrição e validação de todos os processos e respetivas classes de dados foi necessário relacionar os mesmos para, assim, definirmos a Arquitetura de Informação que permitiu analisar a situação da organização, servindo de base a um diagnóstico estruturado dos constrangimentos e dificuldades existentes, bem como futuros.

Assim, construímos uma matriz contendo os processos no eixo vertical e as classes de dados no eixo horizontal. Nas células utilizámos a letra ' $\mathrm{C}$ ' (cria) para indicar as classes de dados criadas pelos processos, e a letra 'U' (usa) para indicar os processos que usam as classes de dados.

De modo a determinar o diagrama de fluxos da Arquitetura de Informação, organizaram-se os processos de modo a que aqueles que tinham muita partilha de dados ficassem próximos. De seguida, reorganizaram-se as classes de dados de modo que a mais próxima do eixo dos processos fosse criada pelo primeiro processo listado, a seguinte (mais próxima) pelo segundo processo e assim consecutivamente. 
RISTI

Revista Ibérica de Sistemas e Tecnologias de Informação

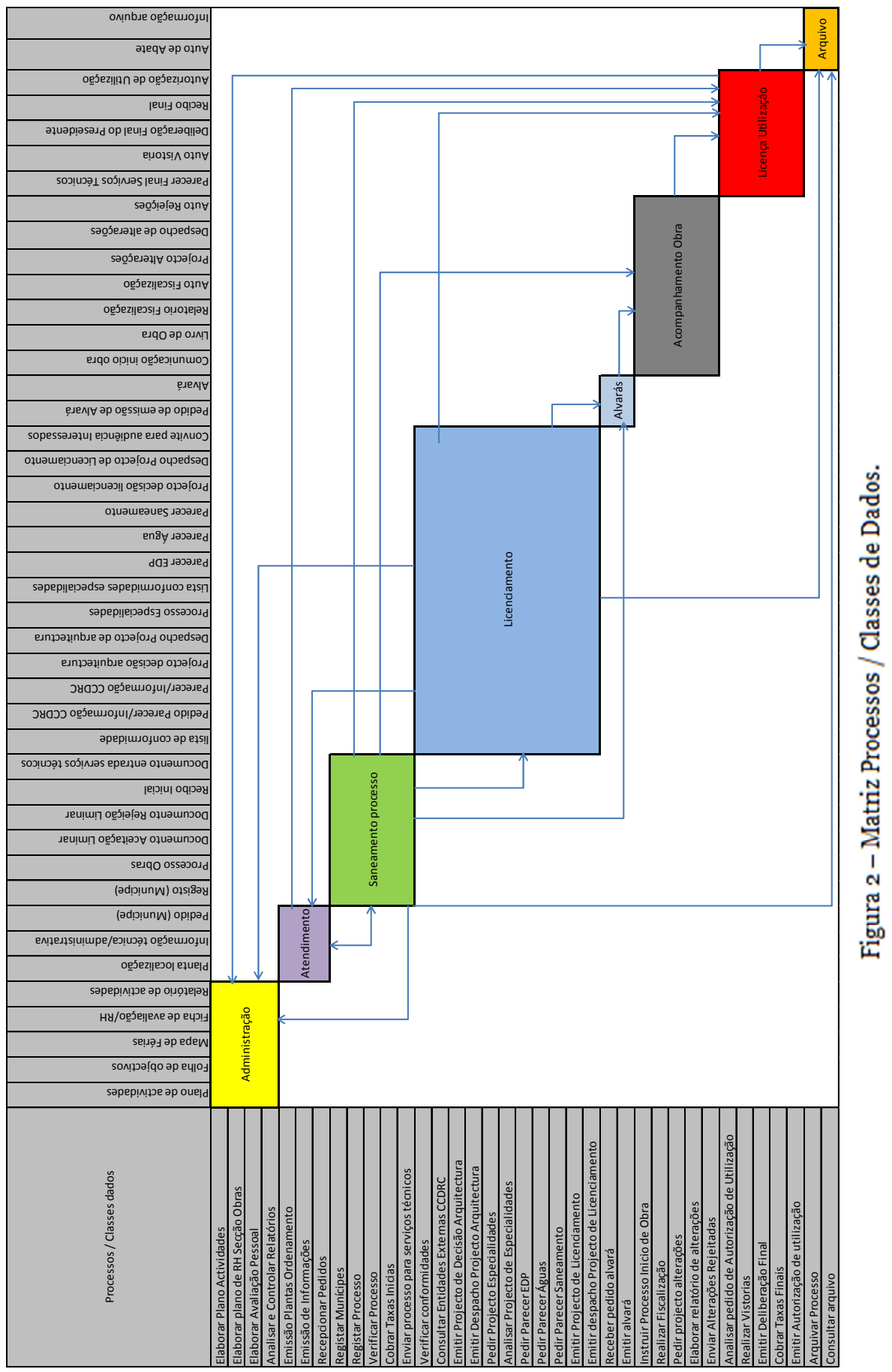


Após a reorganização das linhas e das colunas na matriz, foi possível definir os grupos de processos. Identificados esses grupos, foi possível definir o fluxo de dados entre eles, com base nas classes de dados que cada processo usa.

Na Figura 2 pode ser observada a Matriz Processos/Classes de Dados, tendo sido atribuídos nomes aos grupos dos processos de acordo com a sua função:

- Administração - Grupo de processos e classes de dados referentes à gestão e política da própria Secção de Obras;

- Atendimento - Grupo de processos e classes de dados referentes ao atendimento ao público e instrução inicial de processos;

- Saneamento - Grupo de processos e classes de dados que elabora uma primeira análise de um processo de obras por parte dos serviços administrativos;

- Licenciamento - Grupo de processos e classes de dados que analisam tecnicamente um processo de obras;

- Alvará - Grupo de processos e classes de dados que emite um alvará;

- Acompanhamento Obra - Grupo de processos e classes de dados que realiza o acompanhamento e fiscalização de obras de um munícipe;

- Licença Utilização - Grupo de processos e classes de dados que emite um licença de utilização;

- Arquivo - Grupo de processos e classes de dados que gere o arquivo de processo de obras.

Na Figura 2 também é possível observar o fluxo de dados entre os grupos de processos. Estes fluxos foram criados sempre que um processo de um grupo de processos utilizava uma classe de dados criada por outro processo de outro grupo de processos.

\section{Análise do apoio de TI à Arquitetura de Informação}

Depois de definida a nova arquitetura de informação foi analisado o apoio que recebe atualmente de aplicações informáticas / Tecnologias de Informação (TI). Para esse fim foram elaboradas três matrizes: 1) Matriz Aplicações/Entidades (Figura 3), na qual podemos visualizar que aplicações informáticas apoiam os utilizadores da Secção de Obras; 2) Matriz Aplicações/Processos, na qual podemos visualizar que aplicações apoiam os processos existentes; 3) Matriz Aplicações/Classes de Dados, na qual podem ser visualizadas as aplicações que suportam as classes de dados e assim compreender quais estão atualmente automatizadas e que aplicações mantêm esses dados. 


\begin{tabular}{|c|c|c|c|c|c|c|c|}
\hline Aplicações/Entidades & 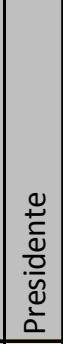 & 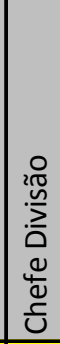 & 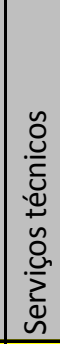 & 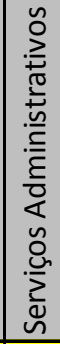 & 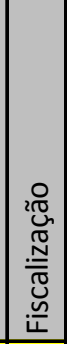 & 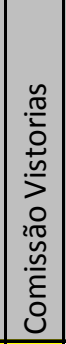 & 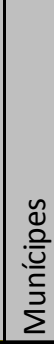 \\
\hline Sistema Processos Obras & A & A & A & A & A & A & \\
\hline Sistema de Taxas & & & & $A$ & & & \\
\hline Sistema de Gestão Documental & A & A & A & A & A & A & $\mathrm{P}$ \\
\hline Sistema Gestão Pessoal & A & A & & & & & \\
\hline Emissão Plantas web & & A & A & A & A & A & $\mathrm{P}$ \\
\hline Portal Autárquico & & A & A & A & & & \\
\hline Autocad & & $A$ & A & A & & & \\
\hline Folha de cálculo & & A & A & A & A & A & A \\
\hline Processamento Texto & A & A & A & A & A & A & A \\
\hline Email & A & A & A & A & A & A & A \\
\hline
\end{tabular}

Figura 3 - Matriz Aplicações/Entidades

\section{Problemas}

Após validação dos passos anteriores foi necessário identificar e definir os principais problemas que surgiram da análise do suporte proporcionado por aplicações informáticas à arquitetura de informação definida e, portanto, do suporte proporcionado aos processos e às classes de dados.

Nesta análise, com recurso a reuniões com os membros das equipas, e com recurso às entrevistas foram identificados e validados os principais problemas. Para sumariá-los foi criada uma tabela com as seguintes colunas: grupo de processos, causa, problema, importância, processo causador, classe-de-dados causadora, e solução sugerida.

Os problemas apesar de surgirem em processos diferentes resultavam em muito da mesma fonte. Os principais problemas são:

1. Falta de informação disponível no sistema (informações em papel por vezes) A Seç̧ão de Obras utiliza principalmente a aplicação SPO (Sistema de Processos de Obras) para os processos de obras e a aplicação SGD (Sistema de Gestão Documental) para registo de correspondência. Estes dois sistemas deviam estar integrados completamente. Paralelamente tanto o SPO como o SGD deviam permitir digitalizar toda a documentação para que a tramitação e análise dos processos fosse sempre baseada em suportes digitais.

2. Excesso de informação lixo - Como nem toda a documentação está digitalizada, e as aplicações não fazem um rastreio da informação, é armazenada informação que não é relevante para as análises dos processos. 
3. Falha na comunicação com os munícipes - A comunicação com os munícipes ainda é feita em papel, o que prejudica os prazos e uma correta tramitação dos documentos. Devia ser integrado nas aplicações SPO e SGD a possibilidade de notificarem e comunicarem digitalmente com os munícipes.

4. Várias aplicações a usarem a mesma informação, sem esta estar partilhada e digitalizada - mais uma vez deve ser integrado o sistema SPO com o sistema SGD para que estes possam usar a mesma informação.

5. Não há um controlo sobre os pedidos externos - A aplicação atual não permite que os pedidos de informações e pareceres externos sejam feitos de forma automática. Como estes pedidos fazem parte da tramitação de um licenciamento, a aplicação SPO deverá ser modificada para efetuar esses pedidos de uma forma automática.

6. Acesso à informação a partir do exterior - Existe uma necessidade, tanto das equipas de fiscalização como das comissões de vistorias, aquando de serviço externo, terem acesso à informação atual dos processos de obras que estão a fiscalizar/vistoriar, pelo que deverá ser implementado um sistema de acesso remoto e em tempo real aos processos existentes no sistema SPO.

\section{Prioridades e recomendações}

Face à informação recolhida e validada, aos problemas identificados, e validação do que é necessário, é imperativo identificar as prioridades e recomendações de desenvolvimento. Para identificação das prioridades utilizámos um método que consiste em identificar e agrupar os critérios em quatro categorias (Figura 4): Benefícios potenciais; Impacto na organização; Probabilidade de sucesso; e Procura. Para cada categoria foi atribuído um peso numa escala de 1 a 10. A soma da pontuação determinou a melhor sequência no desenvolvimento das aplicações informáticas.

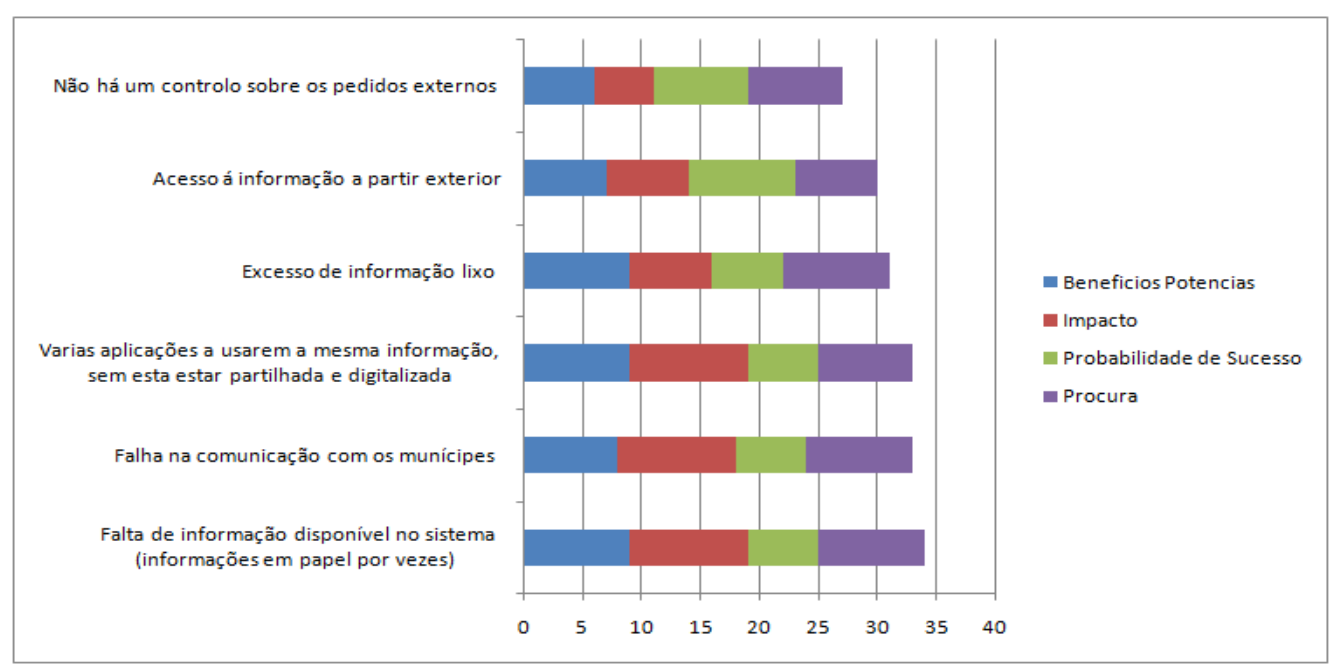

Figura 4 - Definição de prioridades para desenvolvimento 
Face à análise anterior as prioridades para desenvolvimento futuro passam, então, pela correção de:

1. Falta de informação disponível no sistema (informações em papel por vezes);

2. Falha na comunicação com os munícipes;

3. Várias aplicações a usarem a mesma informação, sem esta estar partilhada e digitalizada;

4. Excesso de informação lixo;

5. Acesso à informação a partir do exterior;

6. Não há um controlo sobre os pedidos externos.

Apesar de ter sido identificada a lista inicial de prioridades a desenvolver, esta deve ser reavaliada cada vez que se implementa uma das prioridades. A título de exemplo, no fim da implementação da primeira (Falta de informação disponível no sistema), deve ser feita uma nova identificação de prioridades de desenvolvimento com as restantes.

\section{Discussão e Conclusão}

\subsection{Contributos}

O objetivo principal deste trabalho foi planear uma Arquitetura de Informação para a Secção de Obras Particulares da Câmara Municipal de Penacova. A concretização deste objetivo teve em consideração uma decisão estratégica por parte do Município de Penacova, de forma a alinhar o seu SI com as novas exigências, tanto dos Munícipes que necessitam de melhor acesso à informação e de uma forma mais rápida, como das necessidades legais em garantir respostas mais eficazes e eficientes.

No entanto, foi constatado que um dos grandes problemas com que as Câmaras Municipais se deparam é a má utilização dos sistemas informáticos instalados, associado ao não-alinhamento com as políticas implementadas e desejadas. Outro problema detetado, prende-se com a visão que o poder político tem quando se depara com algumas mudanças. Estas são vistas muitas vezes como um obstáculo e não como um meio para atingir o sucesso.

Felizmente os SI e as TI já começam a ser vistos nas organizações, pelos novos recursos humanos, não somente sob a ótica da tecnologia, mas também como o mote para atingir os objetivos e estratégias. Esta mudança permitiu envolver uma pequena equipa da Câmara Municipal de Penacova para apoiar na aplicação da Metodologia BSP Adaptada, de Amaral \& Varajão (2007), na Secção de Obras Particulares.

Assim, os objetivos específicos passaram por atingir um grau elevado de especialização em sistemas de informação, pelo que foi realizada uma revisão da bibliografia, sobre vários temas da área, e sobre diversas metodologias de planeamento de arquiteturas de informação.

Graças à definição da arquitetura de informação, foi possível a caracterização do sistema de informação, onde podem ser verificadas as aplicações informáticas existentes e qual o apoio que proporcionam à arquitetura de informação definida, onde 
foram identificados e definidos oito grupos de processos (Administração, Atendimento, Saneamento, Licenciamento, Alvarás, Acompanhamento Obra, Licença Utilização e Arquivo) e as quarenta e três classes de dados.

Numa fase posterior, foram identificados os principais problemas, prioridades e recomendações de desenvolvimento futuro. O excesso de informação lixo e a falta de um sistema de gestão documental integrado com os outros sistemas da Câmara Municipal são os principais entraves na implementação e melhoria da arquitetura de informação.

Relativamente à metodologia usada, a Metodologia BSP Adaptada mostrou-se suficientemente flexível e adequada ao contexto onde foi aplicada. Foram, assim, concretizados todos os objetivos propostos no início desta investigação.

\subsection{Limitações e possibilidades de trabalho futuro}

Este trabalho não se esgota nos resultados apresentados neste artigo, sendo que faz parte do planeamento inicial de sistemas de informação da Câmara Municipal de Penacova, em concreto da Secção de Obras Particulares, e deverá ser melhorado e completado se o Município quiser realmente explorar as potencialidades dos SI atuais de forma abrangente e inovadora.

Não deixando de ser um trabalho real e dada a consciência da impossibilidade de explorar com maior detalhe as várias áreas desenvolvidas dentro dos limites temporais e outros, deverá numa parte posterior ser melhorado e estudado as últimas fases da metodologia aplicada.

Na fase final da aplicação da metodologia deverá proceder-se a uma negociação da solução final e verificar se esta se encontra alinhada com os objetivos da Secção de Obras Particulares e da Câmara Municipal.

$\mathrm{Na}$ impossibilidade do Município desenvolver um novo SI que suporte com abrangência a nova arquitetura de informação, é sugerido que deverá disponibilizar e modificar as aplicações existentes, num futuro próximo, em concreto a aplicação SPO e a aplicação SGD, de acordo com a nova arquitetura de informação. Deverá, assim, ser contactada a empresa que desenvolveu estas aplicações para possibilitar a integração das mesmas. A aplicação SGD deverá ser melhorada com a finalidade de permitir a digitalização dos documentos, a tramitação dos processos, o controle dos processos e permitir ainda a comunicação para o exterior.

\section{Referências}

Abbas, A., Mir, S. \& Fereydon, S. (2010), A Method for Benchmarking Application in the Enterprise Architecture Planning Process Based on Federal Enterprise Architecture Framework. Computer Engineering Department, Shahid Beheshti University, Iran.

Almeida, F. B. (2011), e-Government - Análise e Avaliação dos Municípios no Distrito de Viseu. Dissertação de Mestrado, Instituto Politécnico de Viseu, Viseu, Portugal. 
Amaral, L. \& Varajão, J. (2007), Planeamento de Sistemas de Informação. $4^{\mathrm{a}}$ edição, FCA - Editora de Informática, Lisboa, Portugal.

Casagrande, N. G. (2005), Metodologia para Modelagem de Arquitetura de Informação estratégica para pequenas empresas: Uma aplicação no setor de turismo rural. Tese de Doutorado em Administração, Universidade Federal de Santa Catarina, Florianópolis, Brasil.

CIOC (2001), Federal Enterprise Architecture Framework. Version 1.o, Chief Information Officers Council, USA.

Costa, P. (2002), A função Produção de SI/TI - Modelo Informacional. Dissertação de Mestrado, Universidade do Minho, Guimarães, Portugal.

Höst, M. \& Runeson, P. (2007), Checklists for Software Engineering Case Study. Software Engineering Research Group, Lund University, Sweden.

Höst, M. \& Runeson, P. (2008), Guidelines for conducting and reporting case study. Software Engineering Research Group, Lund University, Sweden.

Huang, S. \& Wang, X. (2010), Research on Methods of Integrated Information Systems Based on BSP, Proceedings of The 2010 Fourth International Conference on Genetic and Evolutionary Computing, pp. 546-549. IEEE Computer Society.

IBM. (1984), Business Systems Planning: Information Systems Planning Guide. 4th Ed., International Business Machines Corporation, USA.

Mesquita, A. (2007), Tecnologias e Sistemas de Informação para a indústria da panificação e pastelaria: proposta de uma arquitectura de informação. Dissertação de Mestrado, Universidade de Trás-os-Montes e Alto Douro, Vila Real, Portugal.

Mohammad, D. (2009), A New Methodology For Developing The MIS Master Plan. Review of Business Information Systems, Vol. 13, No. 1, pp. 15-24.

Rocha, A. (2011), Evolution of Information Systems and Technologies Maturity in Healthcare. International Journal of Healthcare Information Systems and Informatics, Vol 6, $\mathrm{N}^{\circ}$ 2, pp. 28-37. DOI: 10.4018/jhisi.2011040103

Rocha, A. \& Santos, P. (2010), Introdução ao Framework de Zachman. Apontamentos de Planeamento de Sistemas de Informação, Universidade Fernando Pessoa, Porto, Portugal.

Rocha, A., Silva, C., Lamas, M., Castro, R. e Silva, S. (2005), Governo Electrónico nas Juntas de Freguesia: Situação na Região do Minho. Actas da $6^{\text {a }}$ Conferência da Associação Portuguesa de Sistemas de Informação. Bragança, Portugal.

Rocha, Á. (2002), O Essencial dos Sistemas de Informação. Apontamentos de Sistemas de Informação, Universidade Fernando Pessoa, Porto, Portugal.

Sakamoto, J. G. \& Ball, F. W. (1982), Supporting Business Systems Planning studies with the DWDC Data Dictionary, IBM System Journal, Vol. 21, No 1, pp. 54-80.

Sayles, A. (2003), Development of Federal Enterprise Architecture Framework using the IBM Rational Unified Process and the Unified Modeling Language. Software Group, IBM, USA. 
Song, H. \& Song, Y. (2010), Enterprise Architecture Institutionalization and Assessment. In Proceedings of the 2010 IEEE/ACIS 9th International Conference on Computer and Information Science (ICIS '10), pp. 870-875.

Souza, J. B. (2001), Uma metodologia para planejamento de arquitetura de informações. Dissertação de Mestrado, Universidade Federal de Santa Catarina, Florianópolis, Brasil.

Spewak, S. H. \& Hill, S. C. (1995), Enterprise Architecture Planning: Developing a Blueprint for Data, Applications, and Technology. John Wiley \& Sons, New York, USA.

Teixeira, P., Brandão, P.L. \& Rocha, A. (2012), Promoting Success in the Introduction of Health Information Systems. International Journal of Enterprise Information Systems, Vol. 8, $\mathrm{N}^{\mathrm{O}}$ 1, pp. 17-27. DOI: 10.4018/jeis.2012010102

Tomé, P. (2004) Modelo de Desenvolvimento de Arquitecturas de Sistemas de Informação. Tese de Doutoramento, Universidade do Minho, Guimarães, Portugal.

Torres, A. (2009), Poder local: Como potenciar a participação dos cidadãos na vida do município de Montijo. Dissertação de Mestrado, ISCTE - Instituto Universitário de Lisboa, Portugal.

Zachman, J. (1987), A Framework for Information Systems Architecture. IBM Systems Journal, Vol. 26, $\mathrm{N}^{\mathrm{o}} 3$, pp. 276-292.

Zachman, J. (1996), The Framework for Enterprise Architecture: Backgroound, Descrition and Utility. Zachman International, USA.

Zachman, J., Inmon, W. \& Geiger, J. (1997), Data Stores, Data Warehousing and the Zachman Framework: Managing Enterprise Knowledge. $1^{\text {st }}$ Edition, McGraw-Hill, New York, USA. 